\title{
Optimal power mean bounds for Yang mean
}

\section{Zhen-Hang Yang ${ }^{1}$, Li-Min Wu² and Yu-Ming Chu ${ }^{*}$}

*Correspondence:

chuyuming2005@126.com

${ }^{1}$ School of mathematics and

Computation Science, Hunan City

University, Yiyang, 413000, China

Full list of author information is

available at the end of the article

\begin{abstract}
In this paper, we prove that the double inequality $M_{p}(a, b)<U(a, b)<M_{q}(a, b)$ holds for all $a, b>0$ with $a \neq b$ if and only if $p \leq 2 \log 2 /(2 \log \pi-\log 2)=0.8684 \cdots$ and $q \geq 4 / 3$, where $U(a, b)$ and $M_{r}(a, b)$ are the Yang and $r$ th power means of $a$ and $b$, respectively.
\end{abstract}

MSC: $26 \mathrm{E} 60$

Keywords: Yang mean; power mean; Neuman-Sándor mean

\section{Introduction}

Let $p \in \mathbb{R}$ and $a, b>0$ with $a \neq b$. Then the $p$ th power mean $M_{p}(a, b)$ of $a$ and $b$ is given by

$$
M_{p}(a, b)=\left(\frac{a^{p}+b^{p}}{2}\right)^{1 / p} \quad(p \neq 0), \quad M_{0}(a, b)=\sqrt{a b}
$$

The main properties for the power mean are given in [1]. It is well known that $M_{p}(a, b)$ is strictly increasing with respect to $p \in \mathbb{R}$ for fixed $a, b>0$ with $a \neq b$. Many classical means are the special cases of the power mean, for example, $M_{-1}(a, b)=2 a b /(a+b)=H(a, b)$ is the harmonic mean, $M_{0}(a, b)=\sqrt{a b}=G(a, b)$ is the geometric mean, $M_{1}(a, b)=(a+b) / 2=$ $A(a, b)$ is the arithmetic mean, and $M_{2}(a, b)=\sqrt{\left(a^{2}+b^{2}\right) / 2}=Q(a, b)$ is the quadratic mean.

Let $L(a, b)=(b-a) /(\log b-\log a), P(a, b)=(a-b) /[2 \arcsin ((a-b) /(a+b))], M(a, b)=$ $(a-b) /\left[2 \sinh ^{-1}((a-b) /(a+b))\right], I(a, b)=\left(a^{a} / b^{b}\right)^{1 /(a-b)} / e$ and $T(a, b)=(a-b) /[2 \arctan ((a-$ $b) /(a+b))]$ be the logarithmic, first Seiffert, Neuman-Sándor, identric, and second Seiffert means of two distinct positive real numbers $a$ and $b$, respectively. Then it is well known that the inequalities

$$
\begin{aligned}
H(a, b) & <G(a, b)<L(a, b)<P(a, b) \\
& <I(a, b)<A(a, b)<M(a, b)<T(a, b)<Q(a, b)
\end{aligned}
$$

hold for all $a, b>0$ with $a \neq b$.

Recently, the bounds for certain bivariate means in terms of the power mean have been the subject of intensive research. Seiffert [2] proved that the inequalities

$$
\frac{2}{\pi} M_{1}(a, b)<P(a, b)<M_{1}(a, b)<T(a, b)<M_{2}(a, b)
$$

hold for all $a, b>0$ with $a \neq b$.

\section{Springer}

๑2014 Yang et al.; licensee Springer. This is an Open Access article distributed under the terms of the Creative Commons Attribution License (http://creativecommons.org/licenses/by/2.0), which permits unrestricted use, distribution, and reproduction in any medium, provided the original work is properly cited. 
Jagers [3] proved that the double inequality

$$
M_{1 / 2}(a, b)<P(a, b)<M_{2 / 3}(a, b)
$$

holds for all $a, b>0$ with $a \neq b$.

In $[4,5]$, Hästö established that

$$
P(a, b)>M_{\log 2 / \log \pi}(a, b), \quad P(a, b)>\frac{2 \sqrt{2}}{\pi} M_{2 / 3}(a, b)
$$

for all $a, b>0$ with $a \neq b$.

Witkowski [6] proved that the double inequality

$$
\frac{2 \sqrt{2}}{\pi} M_{2}(a, b)<T(a, b)<\frac{4}{\pi} M_{1}(a, b)
$$

holds for all $a, b>0$ with $a \neq b$.

In [7], Costin and Toader presented the result that

$$
M_{\log 2 /(\log \pi-\log 2)}(a, b)<T(a, b)<M_{5 / 3}(a, b)
$$

for all $a, b>0$ with $a \neq b$.

$\mathrm{Chu}$ and Long [8] proved that the double inequality

$$
M_{p}(a, b)<M(a, b)<M_{q}(a, b)
$$

holds for all $a, b>0$ with $a \neq b$ if and only if $p \leq \log 2 / \log [2 \log (1+\sqrt{2})]=1.224 \cdots$ and $q \geq 4 / 3$.

The following sharp bounds for the logarithmic and identric means in terms of the power means can be found in the literature [9-16]:

$$
\begin{aligned}
& M_{0}(a, b)<L(a, b)<M_{1 / 3}(a, b), \quad M_{2 / 3}(a, b)<I(a, b)<M_{\log 2}(a, b), \\
& M_{0}(a, b)<L^{1 / 2}(a, b) I^{1 / 2}(a, b)<M_{1 / 2}(a, b), \\
& M_{\log 2 /(1+\log 2)}(a, b)<\frac{L(a, b)+I(a, b)}{2}<M_{1 / 2}(a, b)
\end{aligned}
$$

for all $a, b>0$ with $a \neq b$.

Recently, Yang [17] introduced the Yang mean $U(a, b)$ of two distinct positive real numbers $a$ and $b$ as follows:

$$
U(a, b)=\frac{a-b}{\sqrt{2} \arctan \frac{a-b}{\sqrt{2 a b}}},
$$

and he proved that the inequalities

$$
\begin{aligned}
& P(a, b)<U(a, b)<T(a, b), \quad \frac{G(a, b) T(a, b)}{A(a, b)}<U(a, b)<\frac{P(a, b) Q(a, b)}{A(a, b)}, \\
& Q^{1 / 2}(a, b)\left[\frac{2 G(a, b)+Q(a, b)}{3}\right]^{1 / 2}<U(a, b)<Q^{2 / 3}(a, b)\left[\frac{G(a, b)+Q(a, b)}{2}\right]^{1 / 3},
\end{aligned}
$$




$$
\frac{G(a, b)+Q(a, b)}{2}<U(a, b)<\left[\frac{2}{3}\left(\frac{G(a, b)+Q(a, b)}{2}\right)^{1 / 2}+\frac{1}{3} Q^{1 / 2}(a, b)\right]^{2}
$$

hold for all $a, b>0$ with $a \neq b$.

In [18], Yang et al. presented several sharp bounds for the Yang mean $U(a, b)$ in terms of the geometric mean $G(a, b)$ and quadratic mean $Q(a, b)$.

The main purpose of this article is to find the greatest value $p$ and the least value $q$ such that the double inequality

$$
M_{p}(a, b)<U(a, b)<M_{q}(a, b)
$$

holds for all $a, b>0$ with $a \neq b$.

\section{Lemmas}

In order to prove our main results we need several lemmas, which we present in this section.

Lemma 2.1 Let $f_{1}:(0,1) \times \mathbb{R} \rightarrow \mathbb{R}$ be defined by

$$
f_{1}(x, p)=\frac{\left(1-x^{2}\right)\left(1+x^{p}\right)}{\sqrt{x}\left(1+x^{2}\right)\left(1+x^{p-1}\right)}-\sqrt{2} \arctan \frac{1-x}{\sqrt{2 x}} .
$$

Then

(1) $f_{1}(x, p)$ is strictly decreasing with respect to $x$ on $(0,1)$ if and only if $p \geq 4 / 3$;

(2) $f_{1}(x, p)$ is strictly increasing with respect to $x$ on $(0,1)$ if and only if $p \leq 1 / 2$.

Proof It follows from (2.1) that

$$
\frac{\partial f_{1}(x, p)}{\partial x}=\frac{(1-x) x^{p-1 / 2}}{2\left(1+x^{2}\right)^{2}\left(x+x^{p}\right)^{2}} f_{2}(x, p)
$$

where

$$
\begin{aligned}
f_{2}(x, p)= & x^{1-p}\left(-1+x-5 x^{2}-3 x^{3}\right)+x^{p}\left(3+5 x-x^{2}+x^{3}\right)-(2 p-1) \\
& +4 x-4 x^{3}+(2 p-1) x^{4}
\end{aligned}
$$

(1) If $f_{1}(x, p)$ is strictly decreasing with respect to $x$ on $(0,1)$, then $(2.2)$ leads to the conclusion that $f_{2}(x, p)<0$ for all $x \in(0,1)$. In particular, from (2.3) we have

$$
\lim _{x \rightarrow 1^{-}} \frac{f_{2}(x, p)}{1-x}=-24\left(p-\frac{4}{3}\right) \leq 0 \text {. }
$$

Therefore, $p \geq 4 / 3$ follows from (2.4).

If $p \geq 4 / 3$, then it follows from (2.3) that

$$
\begin{aligned}
\frac{\partial f_{2}(x, p)}{\partial p}= & {\left[x^{p}\left(x^{3}-x^{2}+5 x+3\right)+x^{1-p}\left(3 x^{3}+5 x^{2}-x+1\right)\right] \log x } \\
& -2\left(1-x^{4}\right)<0
\end{aligned}
$$

for all $x \in(0,1)$. 
Equation (2.3) and inequality (2.5) lead to the conclusion that

$$
\begin{aligned}
f_{2}(x, p) \leq & f_{2}\left(x, \frac{4}{3}\right)=-\frac{x^{-1 / 3}}{3}\left(1-x^{2 / 3}\right)^{3} \\
& \times\left(3 x^{8 / 3}+5 x^{7 / 3}+9 x^{2}+12 x^{5 / 3}+6 x^{4 / 3}+12 x+9 x^{2 / 3}+5 x^{1 / 3}+3\right)<0
\end{aligned}
$$

for all $x \in(0,1)$.

Therefore, $f_{1}(x, p)$ is strictly decreasing with respect to $x$ on $(0,1)$ follows from $(2.2)$ and (2.6).

(2) If $f_{1}(x, p)$ is strictly increasing with respect to $x$ on $(0,1)$, then $(2.2)$ leads to the conclusion that $f_{2}(x, p)>0$ for all $x \in(0,1)$. In particular, we have $f_{2}\left(0^{+}, p\right) \geq 0$ and we assert that $p \leq 1 / 2$. Indeed, from (2.3) we clearly see that $f_{2}\left(0^{+}, p\right)=-\infty$ for $p>1, f_{2}\left(0^{+}, 1\right)=-2$, $f_{2}\left(0^{+}, 0\right)=4, f_{2}\left(0^{+}, p\right)=\infty$ for $p<0$, and $f_{2}\left(0^{+}, p\right)=1-2 p$ for $0<p<1$.

If $p \leq 1 / 2$, then inequality (2.5) holds again. It follows from (2.3) and (2.5) that

$$
f_{2}(x, p) \geq f_{2}\left(x, \frac{1}{2}\right)=2 x^{1 / 2}(1-x)\left(x^{2}+2 x^{3 / 2}+4 x+2 x^{1 / 2}+1\right)>0
$$

for all $x \in(0,1)$.

Therefore, $f_{1}(x, p)$ is strictly increasing with respect to $x$ on $(0,1)$ follows from $(2.2)$ and (2.7).

Lemma 2.2 Let $f_{1}:(0,1) \times \mathbb{R} \rightarrow \mathbb{R}$ be defined by (2.1). Then

(1) $f_{1}(x, p)>0$ for all $x \in(0,1)$ if and only if $p \geq 4 / 3$;

(2) $f_{1}(x, p)<0$ for all $x \in(0,1)$ if and only if $p \leq 1 / 2$.

Proof (1) If $f_{1}(x, p)>0$ for all $x \in(0,1)$, then from (2.1) and the L'Hôpital rules we have

$$
\lim _{x \rightarrow 1^{-}} \frac{f_{1}(x, p)}{(1-x)^{3}}=\frac{1}{12}(3 p-4) \geq 0
$$

and $p \geq 4 / 3$.

If $p \geq 4 / 3$, then (2.1) and Lemma 2.1(1) lead to the conclusion that $f_{1}(x, p)>f_{1}(1, p)=0$ for all $x \in(0,1)$.

(2) If $f_{1}(x, p)<0$ for all $x \in(0,1)$, then $f_{1}\left(0^{+}, p\right) \leq 0$. We claim that $p \leq 1 / 2$. Indeed, it follows from (2.1) that $f_{1}\left(0^{+}, p\right)=+\infty$ if $p>1 / 2$.

If $p \leq 1 / 2$, then (2.1) and Lemma 2.1(2) lead to the conclusion that $f_{1}(x, p)<f_{1}(1, p)=0$ for all $x \in(0,1)$.

Lemma 2.3 Let $f_{3}:(0,1) \times \mathbb{R} \rightarrow \mathbb{R}$ be defined by

$$
\begin{aligned}
f_{3}(x, p)= & -x^{1-2 p}+x^{2-2 p}-5 x^{3-2 p}-3 x^{4-2 p}+3+5 x-x^{2}+x^{3} \\
& -(2 p-1) x^{-p}+4 x^{1-p}-4 x^{3-p}+(2 p-1) x^{4-p}
\end{aligned}
$$

Then $\partial^{4} f_{3}(x, p) / \partial x^{4}<0$ for all $x \in(0,1)$ if $p \in(1,4 / 3)$. 
Proof It follows (2.8) that

$$
x^{p+4} \frac{\partial^{4} f_{3}(x, p)}{\partial x^{4}}=x^{1-p}\left(a_{3} x^{3}+a_{2} x^{2}+a_{1} x+a_{0}\right)+b_{4} x^{4}+b_{3} x^{3}+b_{1} x+b_{0}
$$

where

$$
\begin{aligned}
& a_{3}=-3(2 p-1)(2 p-2)(2 p-3)(2 p-4)<0, \\
& a_{2}=-10 p(2 p-1)(2 p-2)(2 p-3)>0, \\
& a_{1}=2 p(2 p-1)(2 p+1)(2 p-2)>0, \\
& a_{0}=-2 p(2 p-1)(2 p+1)(2 p+2)<0, \\
& b_{4}=(2 p-1)(p-1)(p-2)(p-3)(p-4)<0, \\
& b_{3}=-4 p(p-1)(p-2)(p-3)<0, \\
& b_{1}=4 p(p-1)(p+1)(p+2)>0, \\
& b_{0}=-p(2 p-1)(p+1)(p+2)(p+3)<0 .
\end{aligned}
$$

From (2.11)-(2.13) and (2.16) together with (2.17) we get

$$
\begin{aligned}
& a_{2} x^{2}+a_{1} x+a_{0}<a_{2}+a_{1}+a_{0}=-4 p(2 p-1)\left(10 p^{2}-21 p+17\right)<0, \\
& b_{1} x+b_{0}<b_{1}+b_{0}=-p(p+2)(p+1)\left(2 p^{2}+p+1\right)<0
\end{aligned}
$$

for all $x \in(0,1)$.

Therefore, Lemma 2.3 follows easily from (2.9), (2.10), (2.14), (2.15), (2.18), and (2.19).

Lemma 2.4 Let $f_{3}:(0,1) \times \mathbb{R} \rightarrow \mathbb{R}$ be defined by (2.8). Then $\partial^{2} f_{3}(x, p) / \partial x^{2}<0$ for all $x \in$ $(0,1)$ if $p \in(1 / 2,4 / 3)$.

Proof It follows from (2.8) that

$$
\begin{aligned}
x^{p+2} \frac{\partial^{2} f_{3}(x, p)}{\partial x^{2}}= & 6 x^{p+3}+(2 p-1)(p-3)(p-4) x^{4}-2 x^{p+2} \\
& -3(2 p-3)(2 p-4) x^{4-p}-5(2 p-2)(2 p-3) x^{3-p} \\
& +(2 p-1)(2 p-2) x^{2-p}-2 p(2 p-1) x^{1-p} \\
& -4(p-2)(p-3) x^{3}+4 p(p-1) x-p(2 p-1)(p+1), \\
\left.\frac{\partial^{2} f_{3}(x, p)}{\partial x^{2}}\right|_{x=1}= & -48\left(\frac{4}{3}-p\right)\left(\frac{3}{2}-p\right)<0, \\
\left.\frac{\partial^{3} f_{3}(x, p)}{\partial x^{3}}\right|_{x=1}= & 88 p^{3}-300 p^{2}+380 p-144 .
\end{aligned}
$$

We divide the proof into two cases.

Case 1. $p \in(1 / 2,1]$. Then from

$$
(2 p-1)(p-3)(p-4)>0, \quad-3(2 p-3)(2 p-4)<0, \quad-5(2 p-2)(2 p-3) \leq 0,
$$




$$
\begin{aligned}
& (2 p-1)(2 p-2) \leq 0, \quad-2 p(2 p-1)<0, \quad-4(p-2)(p-3)<0, \\
& 4 p(p-1)<0, \quad-p(2 p-1)(p+1)<0, \\
& 0<x^{4} \leq x^{p+3}<x^{4-p} \leq x^{3} \leq x^{p+2}<x^{3-p} \leq x^{2}<x^{2-p} \leq x<x^{1-p} \leq 1
\end{aligned}
$$

and (2.20) we clearly see that

$$
\begin{aligned}
x^{p+2} \frac{\partial^{2} f_{3}(x, p)}{\partial x^{2}}< & {[6+(2 p-1)(p-3)(p-4)] x^{4-p}+[-2-3(2 p-3)(2 p-4)} \\
& -5(2 p-2)(2 p-3)+(2 p-1)(2 p-2)-2 p(2 p-1) \\
& -4(p-2)(p-3)+4 p(p-1) \\
& -p(2 p-1)(p+1)] x^{4-p} \\
= & -8(3 p-4)(2 p-3) x^{4-p}<0
\end{aligned}
$$

for all $x \in(0,1)$.

Case 2. $p \in(1,4 / 3]$. Then (2.22) leads to

$$
\left.\frac{\partial^{3} f_{3}(x, p)}{\partial x^{3}}\right|_{x=1}=88(p-1)\left(p-\frac{53}{44}\right)^{2}+\frac{887}{22}(p-1)+24>0
$$

It follows from Lemma 2.3 and $(2.23)$ that $\partial^{2} f_{3}(x, p) / \partial x^{2}$ is strictly increasing with respect to $x$ on $(0,1)$.

Therefore, $\partial^{2} f_{3}(x, p) / \partial x^{2}<0$ for all $x \in(0,1)$ follows from $(2.21)$ and the monotonicity of the $\partial^{2} f_{3}(x, p) / \partial x^{2}$ with respect to $x$ on the interval $(0,1)$.

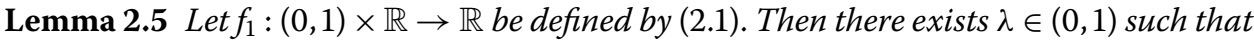
$f_{1}(x, p)$ is strictly decreasing with respect to $x$ on the interval $(0, \lambda]$ and strictly increasing with respect to $x$ on the interval $[\lambda, 1)$ if $p \in(1 / 2,4 / 3)$.

Proof Let $f_{2}(x, p)$ and $f_{3}(x, p)$ be defined by (2.3) and (2.8), respectively. Then from (2.8) we clearly see that

$$
\begin{aligned}
& f_{3}(1, p)=0, \quad f_{3}\left(0^{+}, p\right)=-\infty, \\
& \left.\frac{\partial f_{3}(x, p)}{\partial x}\right|_{x=1}=8(3 p-4)<0, \quad \lim _{x \rightarrow 0^{+}} \frac{\partial f_{3}(x, p)}{\partial x}=+\infty .
\end{aligned}
$$

It follows from Lemma 2.4 and (2.25) that there exists $\lambda_{0} \in(0,1)$ such that $f_{3}(x, p)$ is strictly increasing with respect to $x$ on $\left(0, \lambda_{0}\right]$ and strictly decreasing with respect to $x$ on $\left[\lambda_{0}, 1\right)$. This in conjunction with $(2.24)$ leads to the conclusion that there exists $\lambda \in(0,1)$ such that $f_{3}(x, p)<0$ for $x \in(0, \lambda)$ and $f_{3}(x, p)>0$ for $x \in(\lambda, 1)$.

Note that

$$
f_{2}(x, p)=x^{p} f_{3}(x, p)
$$

Therefore, Lemma 2.5 follows from (2.2) and (2.26) together with the piecewise positive and negative of $f_{3}(x, p)$ on $(0,1)$. 
Lemma 2.6 Let $f:(0,1) \times \mathbb{R} \rightarrow \mathbb{R}$ be defined by

$$
\begin{aligned}
& f(x, p)=\log \frac{U(1, x)}{M_{p}(1, x)}=\log \frac{1-x}{\sqrt{2} \arctan \frac{1-x}{\sqrt{2 x}}}-\frac{1}{p} \log \frac{1+x^{p}}{2} \quad(p \neq 0), \\
& f(x, 0)=\lim _{p \rightarrow 0} \log \frac{U(1, x)}{M_{p}(1, x)}=\log \frac{1-x}{\sqrt{2} \arctan \frac{1-x}{\sqrt{2 x}}}-\frac{1}{2} \log x .
\end{aligned}
$$

Then the following statements are true:

(1) $f(x, p)$ is strictly increasing with respect to $x$ on $(0,1)$ if and only if $p \geq 4 / 3$;

(2) $f(x, p)$ is strictly decreasing with respect to $x$ on $(0,1)$ if and only if $p \leq 1 / 2$;

(3) If $1 / 2<p<4 / 3$, then there exists $\mu \in(0,1)$ such that $f(x, p)$ is strictly increasing with respect to $x$ on $(0, \mu]$ and strictly decreasing with respect to $x$ on $[\mu, 1)$.

Proof It follows from (2.27) and (2.28) that

$$
\frac{\partial f(x, p)}{\partial x}=\frac{1+x^{p-1}}{\sqrt{2}(1-x)\left(1+x^{p}\right) \arctan \frac{1-x}{\sqrt{2 x}}} f_{1}(x, p),
$$

where $f_{1}(x, p)$ is defined by $(2.1)$.

Therefore, parts (1) and (2) follow from Lemma 2.2 and (2.29).

Next, we prove part (3). If $1 / 2<p<4 / 3$, then (2.1) leads to

$$
f_{1}\left(0^{+}, p\right)=+\infty, \quad f_{1}(1, p)=0 .
$$

From Lemma 2.5 and $(2.30)$ we clearly see that there exists $\mu \in(0,1)$ such that $f_{1}(x, p)>0$ for $x \in(0, \mu)$ and $f_{1}(x, p)<0$ for $x \in(\mu, 1)$.

Therefore, part (3) follows from (2.29) and the fact that $f_{1}(x, p)>0$ for $x \in(0, \mu)$ and $f_{1}(x, p)<0$ for $x \in(\mu, 1)$.

\section{Main results}

\section{Theorem 3.1 The double inequality}

$$
M_{p}(a, b)<U(a, b)<M_{q}(a, b)
$$

holds for all $a, b>0$ with $a \neq b$ if and only if $p \leq p_{0}=2 \log 2 /(2 \log \pi-\log 2)=0.8684 \cdots$ and $q \geq 4 / 3$.

Proof Since both the Yang mean $U(a, b)$ and the $r$ th power mean $M_{r}(a, b)$ are symmetric and homogeneous of degree 1 , without loss of generality, we assume that $a=1$ and $b=x \in$ $(0,1)$.

We first prove that the inequality $U(1, x)<M_{q}(1, x)$ holds for all $x \in(0,1)$ if and only if $q \geq 4 / 3$.

If $q=4 / 3$, then from (2.27) and Lemma 2.6(1) we get

$$
\log \frac{U(1, x)}{M_{4 / 3}(1, x)}=f\left(x, \frac{4}{3}\right)<f\left(1^{-}, \frac{4}{3}\right)=0
$$

for all $x \in(0,1)$. 
Therefore, $U(1, x)<M_{q}(1, x)$ for all $x \in(0,1)$ and $q \geq 4 / 3$ follows from (3.1) and the monotonicity of the function $q \rightarrow M_{q}(1, x)$.

If $U(1, x)<M_{q}(1, x)$, then $(2.27)$ and $(2.28)$ lead to $f(x, q)<0$ for all $x \in(0,1)$. In particular, we have

$$
\lim _{x \rightarrow 1^{-}} \frac{f(x, q)}{(1-x)^{2}}=\frac{1}{8}\left(\frac{4}{3}-q\right) \leq 0
$$

and $q \geq 4 / 3$.

Next, we prove that the inequality $U(1, x)>M_{p}(1, x)$ holds for all $x \in(0,1)$ if and only if $p \leq p_{0}$.

If $U(1, x)>M_{p}(1, x)$ holds for all $x \in(0,1)$, then (2.27) leads to $f(x, p)>0$ for all $x \in(0,1)$. In particular, we have

$$
f\left(0^{+}, p\right)=\left(\frac{1}{p}+\frac{1}{2}\right) \log 2-\log \pi \geq 0 .
$$

We claim that $p \leq p_{0}$. Indeed, $p \leq p_{0}$ follows from (3.2) if $p>0$, and $p<p_{0}$ is obvious if $p<0$.

If $p=p_{0}$, then (2.27) leads to

$$
f\left(0^{+}, p_{0}\right)=f\left(1, p_{0}\right)=0 .
$$

It follows from (2.27) and (3.3) together with Lemma 2.6(3) that

$$
\log \frac{U(1, x)}{M_{p_{0}}(1, x)}=f\left(x, p_{0}\right)>0
$$

for all $x \in(0,1)$.

Therefore, $U(1, x)>M_{p}(1, x)$ for all $x \in(0,1)$ and $p \leq p_{0}$ follows from (3.4) and the monotonicity of the function $p \rightarrow M_{p}(1, x)$.

Theorem 3.2 Let $a, b>0$ with $a \neq b$. Then the double inequality

$$
\frac{2^{5 / 4}}{\pi} M_{4 / 3}(a, b)<U(a, b)<\frac{2^{5 / 2}}{\pi} M_{1 / 2}(a, b)
$$

holds with the best possible constants $2^{5 / 4} / \pi$ and $2^{5 / 2} / \pi$.

Proof It follows from Lemma 2.6(1) and (2) together with (2.27) that

$$
\log \frac{U(1, x)}{M_{1 / 2}(1, x)}=f\left(x, \frac{1}{2}\right)<f\left(0^{+}, \frac{1}{2}\right)=\log \frac{2^{5 / 2}}{\pi}
$$

and

$$
\log \frac{U(1, x)}{M_{4 / 3}(1, x)}=f\left(x, \frac{4}{3}\right)>f\left(0^{+}, \frac{4}{3}\right)=\log \frac{2^{5 / 4}}{\pi}
$$

for all $x \in(0,1)$. 
Therefore, $2^{5 / 4} / \pi M_{4 / 3}(1, x)<U(1, x)<2^{5 / 2} / \pi M_{1 / 2}(1, x)$ for all $x \in(0,1)$ follows from (3.5) and (3.6), and the optimality of the parameters $2^{5 / 4} / \pi$ and $2^{5 / 2} / \pi$ follows from the monotonicity of the functions $f(x, 1 / 2)$ and $f(x, 4 / 3)$.

Remark 3.1 For all $a_{1}, a_{2}, b_{1}, b_{2}>0$ with $a_{1} / b_{1}<a_{2} / b_{2}<1$. Then from Lemma 2.6(1) and (2) together with (2.27) we clearly see that the Ky Fan type inequalities

$$
\frac{M_{p}\left(a_{2}, b_{2}\right)}{M_{p}\left(a_{1}, b_{1}\right)}<\frac{U\left(a_{2}, b_{2}\right)}{U\left(a_{1}, b_{1}\right)}<\frac{M_{q}\left(a_{2}, b_{2}\right)}{M_{q}\left(a_{1}, b_{1}\right)}
$$

hold if and only if $p \geq 4 / 3$ and $q \leq 1 / 2$.

Let $p \in \mathbb{R}$ and $L_{p}(a, b)=\left(a^{p+1}+b^{p+1}\right) /\left(a^{p}+b^{p}\right)$ be the $p$ th Lehmer mean of two positive real numbers and $a$ and $b$. Then the function $f_{1}(x, p)$ defined by (2.1) can be rewritten as

$$
f_{1}(x, p)=(1-x)\left[\frac{A(1, x) L_{p-1}(1, x)}{G(1, x) Q^{2}(1, x)}-\frac{1}{U(1, x)}\right]
$$

From Lemma 2.2 and (3.7) we get Remark 3.2.

Remark 3.2 The double inequality

$$
\frac{G(a, b) Q^{2}(a, b)}{A(a, b) L_{p-1}(a, b)}<U(a, b)<\frac{G(a, b) Q^{2}(a, b)}{A(a, b) L_{q-1}(a, b)}
$$

holds for all $a, b>0$ with $a \neq b$ if and only if $p \geq 4 / 3$ and $q \leq 1 / 2$.

\section{Competing interests}

The authors declare that they have no competing interests.

\section{Authors' contributions}

Z-HY provided the main idea and carried out the proof of Lemmas 2.1 and 2.2. L-MW carried out the proof of

Lemmas 2.3-2.6. Y-MC carried out the proof of Theorems 3.1 and 3.2. All authors read and approved the final manuscript.

\section{Author details}

${ }^{1}$ School of mathematics and Computation Science, Hunan City University, Yiyang, 413000, China. ${ }^{2}$ Department of Mathematics, Huzhou University, Huzhou, 313000, China.

\section{Acknowledgements}

This research was supported by the Natural Science Foundation of China under Grants 11171307 and 61374086, and the Natural Science Foundation of Zhejiang Province under Grant LY13A010004.

\section{Received: 23 June 2014 Accepted: 25 September 2014 Published: 16 Oct 2014}

\section{References}

1. Bullen, PS, Mitrinović, DS, Vasić, PM: Means and Their Inequalities. Reidel, Dordrecht (1988)

2. Seiffert, HJ: Aufgabe $\beta$ 16. Ginkgo-Wurzel 29, 221-222 (1995)

3. Jagers, AA: Solution of problem 887. Nieuw Arch. Wiskd. 12, 230-231 (1994)

4. Hästö, PA: A monotonicity property of ratios of symmetric homogeneous means. JIPAM. J. Inequal. Pure Appl. Math. 3(5), Article 71 (2002)

5. Hästö, PA: Optimal inequalities between Seiffert's mean and power mean. Math. Inequal. Appl. 7(1), 47-53 (2004)

6. Witkowski, A: Interpolations of Schwab-Borchardt mean. Math. Inequal. Appl. 16(1), 193-206 (2013)

7. Costin, I, Toader, G: Optimal evaluations of some Seiffert-type means by power means. Appl. Math. Comput. 219(9), 4745-4754 (2013)

8. Chu, Y-M, Long, B-Y: Bounds of the Neuman-Sándor mean using power and identric means. Abstr. Appl. Anal. 2013, Article ID 832591 (2013)

9. Alzer, H: Ungleichungen für Mittelwerte. Arch. Math. 47(5), 422-426 (1986)

10. Alzer, H: Ungleichungen für $(e / a)^{a}(b / e)^{b}$. Elem. Math. 40, 120-123 (1985) 
11. Burk, F: The geometric, logarithmic, and arithmetic mean inequality. Am. Math. Mon. 94(6), 527-528 (1987)

12. Lin, TP: The power mean and the logarithmic mean. Am. Math. Mon. 81, 879-883 (1974)

13. Pittenger, AO: Inequalities between arithmetic and logarithmic means. Publ. Elektroteh. Fak. Univ. Beogr., Ser. Mat. Fiz. 678(715), 15-18 (1980)

14. Pittenger, AO: The symmetric, logarithmic and power means. Publ. Elektroteh. Fak. Univ. Beogr., Ser. Mat. Fiz. 678(715), 19-23 (1980)

15. Stolarsky, KB: The power and generalized logarithmic means. Am. Math. Mon. 87(7), 545-548 (1980)

16. Alzer, H, Qiu, S-L: Inequalities for means in two variables. Arch. Math. 80(2), 201-215 (2003)

17. Yang, Z-H: Three families of two-parameter means constructed by trigonometric functions. J. Inequal. Appl. 2013, Article ID 541 (2013)

18. Yang, Z-H, Chu, Y-M, Song, Y-Q, Li, Y-M: A sharp double inequality for trigonometric functions and its applications. Abstr. Appl. Anal. 2014, Article ID 592085 (2014)

10.1186/1029-242X-2014-401

Cite this article as: Yang et al.: Optimal power mean bounds for Yang mean. Journal of Inequalities and Applications 2014, 2014:401

\section{Submit your manuscript to a SpringerOpen ${ }^{\circ}$ journal and benefit from:}

- Convenient online submission

- Rigorous peer review

- Immediate publication on acceptance

- Open access: articles freely available online

- High visibility within the field

- Retaining the copyright to your article 